\title{
No association between colon cancer and adenocarcinoma of the oesophagus in a population based cohort study in Sweden
}

\author{
J Lagergren, O Nyren
}

\begin{abstract}
Background-Previous reports have indicated an association between Barrett's metaplasia or adenocarcinoma of the oesophagus and colonic neoplasia, but the findings have been inconsistent. If true, such an association suggests common causal mechanisms.

Aims-To test the hypothesis of an association between Barrett's metaplasia or adenocarcinoma of the oesophagus and colonic neoplasia.

Methods-A population based, retrospective cohort study was performed on all Swedish patients with colon cancer diagnosed between 1958 and 1992. 538500 person years at risk were reviewed among the 118030 patients in the cohort (average follow up 4.6 years, median 2.1 years). The standardised incidence ratio (SIR), the ratio of the observed to the expected number of incident oesophageal adenocarcinomas, was used as a measure of relative risk. The expected number was derived from the entire Swedish population.

Results-Eleven oesophageal adenocarcinomas were found during follow up in the cohort, as against 9.5 expected (SIR=1.2; 95\% confidence interval 0.6-2.1). Analysis by latency intervals after diagnosis of colon cancer showed no trend towards increasing or decreasing risk over time. There were no important sex differences in relative risk.

Conclusions-Results provide no support for a common link between colon cancer and oesophageal adenocarcinoma. Although the direct relation between colon cancer and Barrett's oesophagus was not looked at, a search for common aetiological factors or genetic defects may not be fruitful. Screening for colonic neoplasia among patients with malignant or premalignant oesophageal mucosa, or vice versa, may not be warranted. (Gut 1999;44:819-821)
\end{abstract}

Keywords: oesophageal cancer; Barrett's metaplasia; colon cancer; adenocarcinoma

The incidence of adenocarcinoma of the oesophagus is rising almost epidemically, especially among white men in the USA and Western Europe, including Sweden. ${ }^{12}$ The reasons for the increase are unclear and are not explained by known risk factors. There is an urgent need for research on the aetiology of this cancer form and its precursors.

Specialised Barrett's oesophagus (intestinal like columnar cell metaplasia in the oesophageal mucosa), is prone to development of adenocarcinoma. ${ }^{3}$ In several case series, ${ }^{4-7}$ Barrett's oesophagus has also been linked to neoplasia of the colon. Controlled studies of the prevalence of polyps or cancer of the colon among people with a prior diagnosis of Barrett's oesophagus, however, have shown contradictory results, ${ }^{8-11}$ and valid epidemiological data are sparse. A finding of a true association between Barrett's oesophagus and colonic neoplasia would provide a reason to search for common causal factors and could indicate the existence of a generalised defect, possibly genetic. One clinical implication of such a finding might be that screening for colon cancer could be recommended in patients with Barrett's oesophagus, or vice versa, as proposed in previous reports. ${ }^{4-7} 11$

In the light of a significant lifetime risk of adenocarcinoma of the oesophagus among patients with specialised Barrett's columnar cell metaplasia, ${ }^{12} 13$ and of the fact that most oesophageal adenocarcinomas are attributed to this metaplasia, ${ }^{3}$ any association between Barrett's metaplasia and colon cancer should be reflected as an increased risk of colon cancer in patients with adenocarcinoma of the oesophagus. Furthermore, if it is assumed that colon cancer development after Barrett's oesophagus is the result not of Barrett's metaplasia per se, but of common underlying risk factors, there should be an excess risk of oesophageal adenocarcinoma among patients with colon cancer. Our aim was to test the hypothesis of such an association between colon cancer and oesophageal adenocarcinoma.

\section{Subjects and methods}

A population based, retrospective cohort study was carried out among all 118030 patients diagnosed with colon cancer (ICD7 $=153$ ) between 1958 and 1992 in the roughly 98\% complete Swedish Cancer Register. ${ }^{14}$ Follow up for subsequent cancers was accomplished through cross linkage within the register. Information needed for correct censoring was obtained by record linkages to the nationwide registers of Emigration and Causes of Deaths. The National Registration Numbers, unique personal identifiers assigned to all Swedish

Abbreviations used in this paper: SIR, standardised incidence ratio. 
Table 1 The cohort

\begin{tabular}{llll}
\hline & Men & Women & Total \\
\hline Number of individuals & 59922 & 58108 & 118030 \\
Mean age at entry (years) & 69.0 & 69.2 & 69.1 \\
Average year of entry & 1977 & 1977 & 1977 \\
Average follow up time (years) & 4.2 & 4.9 & 4.6 \\
Person years at risk & 252330 & 286170 & 538500 \\
\hline
\end{tabular}

residents, were used to ensure correct matching in these linkages. Each patient was followed up until the diagnosis of an adenocarcinoma of the oesophagus (ICD7 $=150$, histological code 096), death, emigration, or end of follow up (31 December 1992), whichever occurred first. No oesophageal adenocarcinomas were detected first at necropsy in the cohort.

At entry, the cohort consisted of 59922 men and 58108 women with a mean age of 69 years in both sexes (table 1). The colon cancer was verified histologically in $93.5 \%$ of the patients. The cohort members were followed up for an average of 4.6 years (median of 2.1 years) and altogether 538500 person years at risk were reviewed in the study.

The standardised incidence ratio (SIR), the ratio of the observed to the expected number of incident cancers, was used as a measure of relative risk. The expected number of oesophageal adenocarcinomas was calculated by multiplying the observed number of person years in the cohort by age, sex, and calendar year specific incidence rates derived from the entire Swedish population. The $95 \%$ confidence interval was calculated under the assumption that the number of observed cancers followed a Poisson distribution. ${ }^{15}$

\section{Results}

Eleven histologically confirmed adenocarcinomas of the oesophagus were detected during follow up in the cohort, as against 9.5 expected $(\mathrm{SIR}=1.2 ; 95 \%$ confidence interval $0.6-2.1$; table 2). Thus, the observed incidence in the cohort was 2.0, compared with the expected 1.8 per 100000 per year. A non-significant excess in the first year after the diagnosis of colon cancer ( $\mathrm{SIR}=2.0 ; 95 \%$ confidence interval $0.4-5.8$ ), based on three observed cases, may be explained by increased medical surveillance resulting in earlier diagnosis of subclinical cancers of the oesophagus. Accordingly, the SIR for the following four years was somewhat below unity. An analysis which excluded all patients who reached an end point during the first year after diagnosis of colon cancer, and all person time that elapsed in the same time period, showed eight cases of adenocarcinoma of the oesophagus versus 8.0 expected
( $\mathrm{SIR}=1.0 ; 95 \%$ confidence interval $0.5-2.0$; table 2). Analysis by latency intervals after colon cancer diagnosis showed no clear trend towards an increasing or decreasing risk over time (table 2). Nine of the adenocarcinomas of the oesophagus occurred in men, compared with 7.4 expected (SIR $=1.2 ; 95 \%$ confidence interval 0.6-2.3), and two were seen in women, compared with 2.1 expected (SIR $=1.0 ; 95 \%$ confidence interval 0.1-3.4).

\section{Discussion}

Our population based nationwide cohort study, the first of its kind to tackle the possible association between colon cancer and adenocarcinoma of the oesophagus, yielded essentially negative results.

Although colon cancer is common in Sweden, oesophageal cancer is rare. The age standardised incidence rates for the Swedish population increased from 25 to 35 per 100000 per year for colon cancer ${ }^{16-18}$ and from 0.6 to 1.3 per 100000 per year for adenocarcinoma of the oesophagus ${ }^{2}$ during the study period. The rarity of oesophageal adenocarcinoma meant that the power to detect a weak association was limited. Despite more than half a million observed person years at risk, the confidence interval shows that we were unable to rule out the possibility of a doubled risk.

Our results are consistent with those of three controlled studies in which endoscopic examinations failed to confirm an excess risk for colorectal adenoma in patients with Barrett's oesophagus. ${ }^{8-10}$ In one of the studies, however, the control group was too small to allow firm conclusions to be drawn. ${ }^{8}$ In the second study, symptomatic patients, who may also have had an excess risk of colon adenoma, served as controls. ${ }^{9}$ In the third, however, the controls were asymptomatic. ${ }^{10}$ Our findings are at odds with several uncontrolled case series, ${ }^{4-7}$ which showed that a high proportion of patients with Barrett's oesophagus developed colon cancer sooner or later. However, the clinically detected cases of Barrett's oesophagus represent the "tip of an iceberg" of a much larger pool of undetected prevalent cases ${ }^{19}$ and there is an apparent risk of selection bias if the insidious symptoms of the developing colonic neoplasia increase the likelihood that Barrett's oesophagus will be diagnosed. The results of a comparison of patients with oesophageal adenocarcinoma and squamous cell carcinoma from nine cancer registers with regard to their lifetime history of colorectal cancer, ${ }^{11}$ showing a significant excess in the former group (but only in men), also contradict our conclusions.

Table 2 Standardised incidence ratio and 95\% confidence interval for adenocarcinoma of the oesophagus among Swedish men and women with colonic cancer, by latency interval

\begin{tabular}{|c|c|c|c|c|c|c|}
\hline & \multicolumn{6}{|c|}{ Latency interval after diagnosis of colonic cancer (years) } \\
\hline & $<1$ & $1-4$ & $5-9$ & $10-34$ & $0-34$ & $1-34$ \\
\hline Number of patients remaining in the cohort & 118030 & 77823 & 40789 & 20022 & 118030 & 77823 \\
\hline Number of person years at risk & 94017 & 116286 & 144132 & 134065 & 538500 & 444483 \\
\hline Number of observed adenocarcinomas of the oesophagus & 3 & 2 & 4 & 2 & 11 & 8 \\
\hline Number of expected adenocarcinomas of the oesophagus & 1.5 & 2.8 & 2.6 & 2.6 & 9.5 & 8.0 \\
\hline Standardised incidence ratio & 2.0 & 0.7 & 1.5 & 0.8 & 1.2 & 1.0 \\
\hline $95 \%$ confidence interval & $0.4-5.8$ & $0.1-2.6$ & $0.4-3.9$ & $0.1-2.8$ & $0.6-2.1$ & $0.4-2.0$ \\
\hline
\end{tabular}


However, any observation of combination of colorectal and oesophageal cancer in that study was conditional on a sufficient length of survival after the colorectal cancer to develop cancer of the oesophagus. Hence, with the case control design approach used in that study, any difference in survival after colon cancer was diagnosed in the subpopulations that generated the oesophageal adenocarcinomas and squamous cell carcinomas, respectively, would show up as a spurious difference in their association with colorectal cancer. Our study, with its strict person year approach, and with the whole population source as reference, circumvents this problem.

The cohort design and the completeness of the cancer register in our study seem to ensure against both selection and ascertainment bias. Although patients with colon cancer, on average, would be likely to have been under closer medical surveillance than the general population which served as a comparison, the alarming local symptoms of oesophageal cancer usually lead to prompt diagnosis whether the patient is under medical surveillance or not. Misclassification of oesophageal adenocarcinoma as squamous cell carcinoma should be of minor quantitative importance as the vast majority of all oesophageal cancers in Sweden are histologically confirmed; this proportion increased from $82 \%$ in 1958 to $91 \%$ in 1978 and $99 \%$ in $1992 . .^{16-18}$ Bias might have arisen if the oesophageal carcinomas were misclassified as secondary manifestations of the preceding colon cancer. Oesophageal metastases are rare, however, and the high frequency of histological confirmation of tumours in Sweden should minimise such bias.

A theoretically desirable design to study if colon cancer is more common in Barrett's oesophagus would be a large cohort study of colon cancer incidence among patients with Barrett's oesophagus. This study design would, however, need a very large number of these patients and a long follow up.

In conclusion, our Swedish data provide no support for the hypothesis of a common link between colon cancer and oesophageal adeno- carcinoma. A search for common aetiological factors or common susceptibility markers may thus not be fruitful. The results of our study do not point to a need for screening for malignant or premalignant oesophageal mucosa in patients with colonic neoplasms, or vice versa.

The authors wish to express their gratitude to Staffan Josefsson for skilful help with programming and to Lars-Erik Hansson and Tryggvi Stefansson for valuable suggestions during the planning of this study.

1 Blot WJ, Devesa SS, Kneller RW, et al. Rising incidence of adenocarcinoma of the oesophagus and gastric cardia. fAMA 1991;265:1287-9.

2 Hansson LE, Sparen P, Nyren O. Increasing incidence in both histological types of oesophageal carcinomas among men in Sweden. Int f Cancer 1993;54:402-7.

3 Kim R, Weissfeld JL, Reynolds JC, et al. Etiology of Barrett's metaplasia and oesophageal adenocarcinoma. Cancer Epidemiol Biomarkers Prev 1997;6:369-77.

4 Sontag SJ, Schnell TG, Chejfec G, et al. Barrett's oesophagus and colonic tumours. Lancet 1985;i:946-9.

5 Robertson DAF, Ayres RCS, Smith CL. Screening for colonic cancer in patients with Barrett's oesophagus. BMF 1989;298:650

6 Kingston RD, Kiff RS, Walsh SH. Barrett's oesophagus and colonic cancer [letter]. Lancet 1988;i:63.

7 Howden CW, Hornung CA. A systematic review of the association between Barrett's oesophagus and colon neoplasms. Am $\mathcal{F}$ Gastroenterol 1995;90:1814-19.

8 Laitikari R, Laippala P, Isolauri J. Barrett's oesophagus is not a risk factor for colonic neoplasia:a case-control study. Ann Med 1995;27:499-502.

9 Cauvin JM, Goldfain D, Le Rhun M, et al. Multicentre procolorectal adenomas. Lancet 1995;346:1391-4

10 Poorman JC, Lieberman DA, Ippoliti AF, et al. The prevalence of colonic neoplasia in patients with Barrett's oesophagus: prospective assessment in patients 50-80 years oesophagus: prospective assessment in pate
old. Am $\mathcal{F}$ Gastroenterol 1997;92:592-6.

11 Vaughan TL, Kiemeney LALM, McKnight B. Colorectal cancer in patients with oesophageal adenocarcinoma. cancer in patients with oesophageal aden.
Cancer Epidemiol Biomarkers Prev 1995;4:93-7.

12 Hameetman W, Tytgat GN, Houthoff HJ, et al. Barrett's oesophagus: development of dysplasia and adenocarcinoma. Gastroenterology 1989;96:1249-56.

13 Robertson CS, Mayberry JF, Nicholson DA, et al. Value of endoscopic surveillance in the detection of neoplastic change in Barrett's oesophagus. Br F Surg 1988;75:760-3.

14 Mattsson B, Rutqvist LE, Wallgren A. Undernotification of diagnosed cancer cases to the Stockholm Cancer Registry. Int $\mathcal{F}$ Epidemiol 1985;14:64-9.

15 Bailar JC III, Ederer F. Significance factors for the ratio of a Poisson variable to its expectation. Biometrics 1964;20:63943.

16 Cancer Registry. Cancer incidence in Sweden 1992. Stockholm: National Board of Health and Welfare, 1995.

7 Cancer Registry. Cancer incidence in Sweden 1958. Stockholm: National Board of Health and Welfare, 1960.

18 Cancer Registry. Cancer incidence in Sweden 1978. Cancer Registry. Cancer incidence in Sweden 1978.
Stockholm: National Board of Health and Welfare, 1982.

19 Cameron AJ, Zinsmeister AR, Ballard DJ, et al. Prevalence of columnar-lined (Barrett's) oesophagus. Gastroenterology 1990;99:918-22. 